\title{
LOS DETERMINANTES DE LA DIVULGACIÓN DE INFORMACIÓN SOBRE RESPONSABILIDAD SOCIAL CORPORATIVA EN EL SECTOR FINANCIERO: EL CASO ESPAÑOL*
}

\author{
ARTURO HARO DE ROSARIO**, FRANCISCO ALARCÓN SENENT*** \& MARÍA DEL CARMEN CABA PÉREZ**** \\ UNIVERSIDAD DE ALMERÍA (ESPAÑA)
}

Recibido/ Received/ Recebido: 21/03/2012 - Aceptado/ Accepted/Aprovado: 30/04/2012

\begin{abstract}
Resumen
Las empresas han optado dar a conocer su compromiso con el desarrollo económico sostenible, como resultado del rechazo a aquellas organizaciones con conductas que atentan contra los derechos humanos o, bien provocan daños medioambientales. En el presente trabajo se realiza un estudio acerca de la divulgación de información sobre responsabilidad social que presentan las entidades de crédito y de los factores que determinan este suministro de información. Los resultados del trabajo muestran que las memorias sobre sostenibilidad de las entidades analizadas contienen un volumen elevado de información, destacando la relativa a aspectos económicos y sociales. La cotización en el mercado de valores, tipo de entidad y la aplicación del marco de recomendaciones GRI3 son las variables que están más relacionadas con la publicación de información sobre responsabilidad social corporativa. Los hallazgos de la investigación son importantes en el marco de la crisis del sector financiero español.
\end{abstract}

Palabras clave: responsabilidad social corporativa, divulgación de información, sector financiero, entidades de crédito

\section{DISCLOSURE DETERMINANTS OF CORPORATE SOCIAL RESPONSIBILITY INFORMATION IN THE SPANISH FINANCIAL INDUSTRY}

\begin{abstract}
Firms have chosen to make known their commitment with sustainable economic development as a result of the rejection of those organizations with behaviors that violate human rights, or cause environmental damage. It's a study about the disclosure of social responsibility presented by credit institutions. We seek to identify the factors that determine the supply of information. The results of the study show that reports on sustainability of the entities analyzed contain a high volume of infor-
\end{abstract}

* Resultado del grupo de investigación Nuevos enfoques en finanzas y sistemas de información empresarial. Realizado mediante la beca del programa de "Incentivos para la formación de personal docente e investigador predoctoral en las Universidades Públicas de Andalucía, en áreas de conocimiento consideradas deficitarias por necesidades institucionales docentes y de investigación" de la Junta de Andalucía (España).

** Becario de investigación del departamento de Dirección y Gestión de Empresas de la Universidad de Almería. Imparte docencia de materias relacionadas con el área de Economía Financiera y Contabilidad. Correo electrónico: arturo.haro@ual.es

*** Diplomado en Ciencias Empresariales y Licenciado en Administración y Dirección de Empresas. Ha cursado un Máster en Contabilidad y Finanzas Corporativas en la Universidad de Almería y está realizando el doctorado. Correo electrónico: fjalarcon_85@ hotmail.com.

**** Doctora en Contabilidad y profesora titular del Departamento de Dirección y Gestión de Empresas de la Universidad de Almería. Imparte docencia de materias relacionadas con el área de Economía Financiera y Contabilidad a nivel de Master y Doctorado. Actualmente es Vicedecana de la facultad de Ciencias Económicas y Empresariales. Correo electrónico: ccaba@ual.es 
mation, on emphasizing the economic and social aspects. Determinants, trading on the stock market, type of entity and implementation of the framework GRI3 are the variables that are more related to the publication of information on corporate social responsibility. The research findings are important in the context of "Spanish financial crisis".

Keywords: Corporate social responsabilty, information disclosure, credit institutions.

\title{
DETERMINANTES DA DIVULGAÇÃO DE RESPONSABILIDADE SOCIAL CORPORATIVA NO SETOR FINANCEIRO ESPANHOL
}

\section{Resumo}

\begin{abstract}
As empresas têm optado por dar a conhecer o seu compromisso com o desenvolvimento econômico sustentável, como resultado da rejeição destas organizações, com comportamentos que violam os direitos humanos, ou causar danos ambientais. Este trabalho apresenta um estudo sobre a divulgação da responsabilidade social apresentados por instituições de crédito. Procuramos identificar os fatores que determinam o fornecimento de informações. Os resultados do estudo mostram que os relatórios sobre sustentabilidade das entidades analisadas contêm um grande volume de informações, em enfatizando os aspectos econômicos e sociais. A listagem na bolsa de valores, tipo de entidade e implementação das GRI3 são as variáveis que estão mais relacionadas com a publicação de informações sobre responsabilidade social corporativa. Os resultados da pesquisa são importantes no contexto de crise "do sector financeiro espanhol"
\end{abstract}

Palavras chave: Responsabilidade social corporativo, divulgação, setor financiero.

Haro, A., Alarcon, F. \& Caba, M. (2012) Los determinantes de la divulgación de información sobre Responsabilidad Social Corporativa en el sector financiero: el caso español. En: Revista de la Facultad de Ciencias Económicas de la Universidad Militar Nueva Granada. rev.fac.cienc.econ, XX (1)

JEL: M39.

\section{Introducción}

En la sociedad actual, gran parte de la ciudadanía está convencida de que las empresas tienen que asumir una serie de compromisos para lograr una convivencia en armonía entre sus actividades licitas, encaminadas a obtención de beneficios así como la creación de valor para sus socios, y los objetivos globales de la sociedad, enraizados en la idea de la solidaridad entre sus miembros, además la sostenibilidad del sistema económico.

Según AECA (2004), podemos interpretar como responsabilidad social corporativa (en adelante RSC) el compromiso voluntario de las empresas con el desarrollo de la sociedad y la preservación del medio ambiente, desde su composición social, y un comportamiento responsable hacia las personas y grupos sociales con los que interactúa. Siendo su objetivo fundamental introducir elementos de dirección y gestión orientados a innovar y mejorar el impacto de las empresas, de forma que éstas generen externalidades socialmente responsables.

En este marco, la información financiera ha demostrado tener importantes carencias para representar la gestión socialmente responsable de las organizaciones (Larrinaga et al., 2002). Por ello, la divulgación de información voluntaria sobre el desempeño en materia de RSC cobra especial interés.

Las entidades que componen el sector financiero han de ser conscientes de su responsabilidad y de las repercusiones sociales y económicas que puede suponer eludirla. Su función económica no ha de limitarse a intermediar flujos gestionando adecua- 
damente riesgos financieros, sino debe ir más allá, tratando de evaluar y gestionar otro tipo de riesgos éticos, sociales y medioambientales, ofreciendo así productos y servicios que contribuyan al desarrollo sostenible, respondiendo al reclamo de una ciudadanía cada vez más preocupada por el destino de su ahorro y de unos accionistas interesados por los impactos económicos de determinadas gestiones y comportamientos. Por lo tanto, se hace necesaria la rendición de cuentas a los grupos de interés mediante la divulgación de su desempeño económico, medioambiental y social.

Partiendo de esta situación, el trabajo se centra en el estudio de la información voluntaria sobre RSC que divulgan estas entidades en España. Para alcanzar esta meta se ha establecido un doble objetivo. El primero de ellos consiste en comprobar el volumen y tipo de información que divulgan a través de sus memorias de RSC las entidades financieras. El segundo es averiguar cuáles son los principales factores que inciden en la divulgación de información sobre RSC en este tipo de entidades.

Con el fin de lograr los objetivos señalados el trabajo se estructura en seis partes. Tras esta introducción, en el segundo apartado se introduce el concepto de la RSC y se expone su desarrollo en el sector financiero. A continuación, en el tercer apartado se describe la metodología de investigación llevada a cabo, que consta de una fase descriptiva en la que se elabora un índice de información divulgada sobre RSC, y una fase explicativa que comprende la selección de variables independientes, un análisis de correlaciones y una regresión lineal múltiple. En el cuarto apartado se especifica el proceso de selección de las entidades que integran la muestra objeto de estudio. En quinto lugar se exponen los resultados de la investigación $y$, por último, figuran las conclusiones, entre las que se pueden destacar la calidad de las memorias sobre sostenibilidad de las cajas de ahorro, quizás por su marcado fin social, y que aunque no hay acuerdo en la literatura existente sobre los determinantes del suministro de información sostenible, en el caso del sector financiero español la cotización en el mercado de valores, el tipo de entidad y aplicación del marco de recomendaciones GRI3 parecen ser las variables in- dependientes más relacionadas con la divulgación de información sobre RSC.

\section{El sector financiero y la Responsabilidad Social Corporativa}

Para comprobar los antecedentes históricos de lo que hoy se denomina como RSC es necesario remontarse varios siglos, concretamente al origen de las organizaciones que conjugaron capital y trabajo para generar riqueza y beneficio (Navarro, 2008).

Aunque desde los primeros intentos de conceptualización de la RSC han aparecido diversas definiciones en las que se considera que las organizaciones han de ofrecer respuestas a una serie de exigencias que van mas allá de las obligaciones técnicas, económicas y legales, alcanzando así unos beneficios sociales (AECA, 2004; Davis, 1973; Davis \& Blomstrom, 1975; McGuire, 1963), la RSC está en continua evolución y no existe consenso sobre su definición. Por tanto, la RSC ha de ser interpretada como un proceso o camino de integración y mejora permanente.

Según la definición que da el Libro Verde de la Comisión Europea $(2001,7)$ se describe como "la integración voluntaria, por parte de las empresas, de las preocupaciones sociales y medioambientales en sus operaciones comerciales y sus relaciones con sus interlocutores". En cualquier caso, en casi todas las definiciones se observa una serie de características comunes, como son la voluntariedad, la integración, la consistencia y la transparencia (Albareda \& Balaguer, 2007).

En cuanto al desarrollo de la RSC, éste se aceleró rápidamente cuando en la década de los 70 entraron en escena las organizaciones internacionales (Organización de las Naciones Unidas, Organización para la Cooperación y el Desarrollo Económico, Organización Internacional del Trabajo, etc.). En 1997, como resultado de la iniciativa conjunta entre la organización no gubernamental estadounidense Coalition for Environmentally Responsable Economies y el Programa de las Naciones Unidas para el Medio Ambiente nace la Global Reporting Initiative (en adelante GRI). 
La GRI se convirtió pronto en una iniciativa ampliamente respaldada por representantes de empresas, organizaciones asesoras no lucrativas, auditoras, sociedades de inversión y sindicatos, siendo su principal cometido el de plantear un marco a partir del cual una determinada organización pueda evaluar y divulgar su desempeño económico, ambiental y social de acuerdo con unos parámetros comunes, rigurosos, útiles para el usuario y de calidad.

Para proporcionar un marco fiable y creíble sobre el que las organizaciones, con independencia de su tamaño, sector o ubicación, pudieran elaborar sus memorias de sostenibilidad, la GRI confeccionó la Guía para la Elaboración de Memorias de Sostenibilidad. En 2006 se publicó la tercera y última versión de la Guía, conocida como GRI3. No obstante, la iniciativa se encuentra trabajando en la cuarta generación de la Guía, cuya publicación está prevista para 2013.

Centrándonos ahora en el sector financiero, los intermediarios financieros, y en especial las entidades de crédito, juegan un papel activo en el desarrollo económico y social, nacional e internacional, al cargar con la responsabilidad de seleccionar proyectos de inversión y consumo, gestionar riesgos y tener el poder de decisión, en la mayoría de los casos, sobre quién accede al capital y, por lo tanto, sobre qué actividades son financiadas por ellos.

No obstante, las entidades de crédito deben ir más allá de su posición de intermediarios y gestores de riesgos de mercado y exigir la evaluación y gestión de otro tipo de riesgos, como son los éticos, sociales y medioambientales. Del mismo modo, han de ofrecer productos y servicios que contribuyan al desarrollo humano y sostenible del planeta, respondiendo de esta forma al reclamo de una ciudadanía cada vez más preocupada por el destino de su ahorro y de unos accionistas más interesados por el impacto económico de determinados comportamientos poco sostenibles.

Sin embargo, estas responsabilidades han sido escasamente tenidas en cuenta por los economistas y las organizaciones internaciones hasta hace pocos años (Tostes, 2008). Aunque parece que poco a poco la industria financiera comienza a ser consciente de ellas y de las repercusiones económicas que pueden suponer eludir cuestiones como, por ejemplo, la lucha contra la pobreza, las desigualdades sociales o el desarrollo sostenible (De la Cuesta, 2006).

Así las cosas, si se interpreta el termino desarrollo como crecimiento sostenible y cohesión social, se obtiene la conclusión de que las entidades financieras tienen una responsabilidad que va más allá del ámbito puramente económico, extendiéndose al ámbito social y medioambiental. Por lo que el papel de dichas entidades no ha de limitarse a distribuir recursos entre agentes, sino que también, han de asumir una serie de riesgos en esas transferencias de fondos a parte de los meramente financieros (éticos, sociales y medioambientales) y tomar decisiones sobre el destino más o menos sostenible del dinero (De la Cuesta, 2006).

En el caso del sistema financiero español la fuerte presencia de entidades de crédito, intervienen más del $95 \%$ de los flujos financieros de la economía, hace que las citadas responsabilidades sociales $y$ ambientes recaigan sobre estas entidades.

La falta de consideración de los riesgos éticos, sociales y medioambientales asociados a la actividad puede derivar en riesgos económicos. De un modo $\mathrm{u}$ otro, puede quedar en entredicho la reputación de la entidad y, en consecuencia, peligrar la obtención de capital en los mercados, si la gestión de fondos se lleva a cabo sin considerar los riegos éticos, sociales y medioambientales de las empresas en las que invierte. A pesar de esto, la estrategia y práctica diaria de las compañías financieras no cuestiona en profundidad, generalmente, los intereses de otros agentes al margen de los accionistas, ni el impacto social y medioambiental de sus políticas.

De otra parte, las malas prácticas llevadas a cabo en este sector, como el fomento de la evasión fiscal mediante la colocación de fondos en paraísos fiscales, la incorrecta aplicación de códigos de conducta dirigidos a la detección de operaciones de blanqueo de dinero, corrupción o sobornos, la ocultación de información, el aumento del valor real de algunos activos suscritos por las entidades o la transmisión del riesgo a terceros a través de diferentes mecanis- 
mos como las instituciones de inversión colectiva, han dado lugar a escándalos financieros en países como Estados Unidos (Enron, World.Com, Tyco, Global Crossing, Xerox, Adelphia, QWest), Francia (caso Vivendi), Alemania (caso Mannesmann), Holanda (Ahold), Italia (caso Parmalat) y, aunque en menor medida, y con otras características, también en España (caso Banesto, Gescartera y Eurobank).

En definitiva, las malas prácticas del sector financiero pueden tener efectos sobre la estabilidad financiera y económica mundial, sobre la eficiencia y la productividad y sobre la credibilidad y seguridad en el sistema financiero, basado en la confianza y en la transparencia. Razones suficientes para que hayan surgido varias directrices, de carácter voluntario, que tratan de prevenir dichas prácticas y corregir las externalidades que estos comportamientos están teniendo en materia social y medioambiental. Entre ellas destaca el Suplemento Sectorial para Servicios Financieros (en adelante FSSS) de la GRI, confeccionado con el fin de que las distintas organizaciones pertenecientes al sector puedan utilizarlo como referencia para medir e informar sobre sus dimensiones económicas, medioambientales y sociales, así como sobre su estructura organizativa, sus actividades, productos y servicios.

El FSSS incluye comentarios adicionales relacionados con el sector financiero, así como indicadores de desempeño específicos para garantizar que los informes de RSC de las compañías de servicios financieros cubren efectivamente los temas clave del sector. El primer FSSS se editó en 2002 y, tras varias fases de desarrollo, la última versión se publicó 2008.

\section{Metodología}

El estudio se compone de dos partes. En primer lugar, un análisis de carácter descriptivo, con el que se examina la información contenida en las memorias de RSC y en segundo lugar, un análisis de tipo explicativo, cuya finalidad consiste en determinar cuáles son los principales factores que inciden en la divulgación de información sobre RSC en las entidades financieras.

El análisis descriptivo pretende medir, elaborando un índice que estará formado por una serie de ítems, el grado en que las instituciones pertenecientes al sector divulgan información sobre RSC y el tipo de información al que prestan una mayor atención en sus memorias.

Con la finalidad de desarrollar el análisis se establecen como ítems los indicadores principales recomendados por la Guía para la Elaboración de Memorias de Sostenibilidad (GRI, 2006) y por el Suplemento Sectorial para Servicios Financieros (GRI, 2008).

La Guía recoge dichos indicadores en 3 dimensiones (económica, ambiental y social), a lo que es necesario añadir una nueva dimensión incorporada por el suplemento sectorial (impacto de productos y servicios). Cada una de estas cuatro dimensiones constituirá un subíndice que, en conjunto, compondrán un índice agregado al que se denominara como índice de responsabilidad (IR). Los ítems empleados para medir el desempeño en cada una de las dimensiones son los siguientes:

- Impacto de productos y servicios: Para medir este enfoque de gestión, especifico del sector de los servicios financieros, se van a emplear 12 ítems (véase tabla 1) sobre el desempeño que las entidades objeto de estudio realizan en torno al impacto de sus productos y servicios.

- La estimación del subíndice relativo a esta dimensión $\left(\mathrm{IR}_{\mathrm{p}}\right)$ se lleva a cabo del siguiente modo (realizándose el resto de estimaciones de forma análoga):

$$
I R_{p}=\frac{\sum_{i=1}^{12} i r_{i}}{12} \times 100
$$

- Dimensión económica: Esta dimensión se construye a partir de un subíndice $\left(\mathrm{IR}_{e}\right)$ formado por un total de 7 ítems que recogen aspectos de carácter económico sobre presencia en el mercado y relativos a la incidencia de impactos económicos indirectos (véase tabla 2).

- Dimensión ambiental: La dimensión ambiental (subíndice $\mathrm{IR}_{\mathrm{a}}$ ) está representada por 17 ítems, los cuales cubren aspectos de diversa índole en materia medioambiental (ver tabla 3). 
Tabla 1. Ítems sobre el impacto de productos y servicios ${ }^{1}$

\begin{tabular}{|c|c|c|}
\hline i & Cód. & Información a divulgar \\
\hline 1 & FS1 & Políticas con aspectos medioambientales y sociales específicos aplicadas a las líneas de negocios \\
\hline 2 & FS2 & Procedimientos para la evaluación y el control de riesgos sociales y medioambientales en las líneas de negocio \\
\hline 3 & FS3 & $\begin{array}{l}\text { Procesos para monitorizar la implementación por parte de los clientes de los requisitos sociales y medioambientales incluidos en contratos } \\
\text { o transacciones }\end{array}$ \\
\hline 4 & FS4 & $\begin{array}{l}\text { Procesos de mejora en competencia de los empleados para implementar políticas y procedimientos medioambientales y sociales aplica- } \\
\text { dos a las líneas de negocio }\end{array}$ \\
\hline 5 & FS5 & $\begin{array}{l}\text { Interacciones con clientes, sociedades participadas o socios empresariales en relación a los riesgos y oportunidades medioambientales } \\
\text { y sociales }\end{array}$ \\
\hline 6 & FS6 & Porcentaje de la cartera para las líneas de negocio según la región, la dimensión y el sector de actividad \\
\hline 7 & FS7 & $\begin{array}{l}\text { Valor monetario de los productos y servicios diseñados para proporcionar un beneficio social especifico para cada línea de negocio des- } \\
\text { glosado según propósito }\end{array}$ \\
\hline 8 & FS8 & $\begin{array}{l}\text { Valor monetario de los productos y servicios diseñados para proporcionar un beneficio medioambiental especifico para cada línea de } \\
\text { negocio desglosado }\end{array}$ \\
\hline 9 & FS9 & $\begin{array}{l}\text { Cobertura y frecuencia de auditorías para evaluar la implementación de políticas medioambientales y sociales y los procedimientos de } \\
\text { evaluación de riesgos }\end{array}$ \\
\hline 10 & FS10 & $\begin{array}{l}\text { Porcentaje y numero de compañías dentro de la cartera de la entidad con las que la organización informante ha interactuado en temas } \\
\text { medioambientales y sociales }\end{array}$ \\
\hline 11 & FS11 & Porcentaje de activos sujetos a controles sociales o medioambientales, tanto positivos como negativos \\
\hline 12 & FS12 & $\begin{array}{l}\text { Políticas de voto en asuntos medioambientales o sociales en participaciones sobre las cuales la organización informante posee derecho } 0 \\
\text { recomendación de voto }\end{array}$ \\
\hline
\end{tabular}

Tabla 2. Ítems del desempeño económico²

\begin{tabular}{|c|c|l|}
\hline i & Cód. & \multicolumn{1}{c|}{ Información a divulgar } \\
\hline 1 & EC1 & Valor económico directo generado y distribuido \\
\hline 2 & EC2 & Consecuencias financieras y otros riesgos y oportunidades para las actividades de la organización debido al cambio climático \\
\hline 3 & EC3 & Cobertura de las obligaciones de la organización debidas a programas de beneficios sociales \\
\hline 4 & EC4 & Ayudas financieras significativas recibidas del gobierno \\
\hline 5 & EC6 & Política, practicas y proporción de gasto correspondientes a proveedores locales en lugares donde se desarrollen operaciones significativas \\
\hline 6 & EC7 & $\begin{array}{l}\text { Procedimientos para la contratación local y proporción de altos directivos procedentes de la comunidad local en lugares donde se desa- } \\
\text { rrollen operaciones }\end{array}$ \\
\hline 7 & EC8 & $\begin{array}{l}\text { Desarrollo e impacto de las inversiones en infraestructuras y los servicios prestados para el beneficio público mediante compromisos } \\
\text { comerciales, pro bono, o en especie }\end{array}$ \\
\hline
\end{tabular}

1 Elaboración propia a partir de FSSS (2008).

2 Elaboración propia a partir de GRI (2006). 
Tabla 3. Ítems del desempeño ambiental ${ }^{3}$

\begin{tabular}{|c|c|c|}
\hline $\mathbf{i}$ & Cód. & Información a divulgar \\
\hline 1 & EN1 & Materiales utilizados, por peso o volumen \\
\hline 2 & EN2 & Porcentaje de los materiales utilizados que son materiales valorizados \\
\hline 3 & EN3 & Consumo directo de energía desglosado por fuentes primarias \\
\hline 4 & EN4 & Consumo indirecto de energía desglosado por fuentes primarias \\
\hline 5 & EN8 & Captación total de agua por fuentes \\
\hline 6 & EN11 & $\begin{array}{l}\text { Descripción de terrenos adyacentes o ubicados en espacios naturales protegidos o de aéreas de alta biodiversidad no protegidas en } \\
\text { propiedad } 0 \text { arrendados }\end{array}$ \\
\hline 7 & EN12 & $\begin{array}{l}\text { Descripción de los impactos más significativos en la biodiversidad en espacios naturales protegidos o en aéreas de alta biodiversidad } \\
\text { no protegidas }\end{array}$ \\
\hline 8 & EN16 & Emisiones totales, directas e indirectas, de gases de efecto invernadero, en peso \\
\hline 9 & EN17 & Otras emisiones indirectas de gases de efecto invernadero, en peso \\
\hline 10 & EN19 & Emisiones de sustancias destructoras de la capa ozono, en peso \\
\hline 11 & EN20 & NO, SO y otras emisiones significativas al aire por tipo y peso \\
\hline 12 & EN21 & Vertidos totales de aguas residuales, según su naturaleza y destino \\
\hline 13 & EN22 & Peso total de residuos gestionados, según tipo y método de tratamiento \\
\hline 14 & EN23 & Número total y volumen de los derrames accidentales más significativos \\
\hline 15 & EN26 & Iniciativas para mitigar los impactos ambientales de los productos y servicios \\
\hline 16 & EN27 & $\begin{array}{l}\text { Porcentaje de productos vendidos, y sus materiales de embalaje, que son recuperados al final de su vida útil, por categorías de } \\
\text { productos }\end{array}$ \\
\hline 17 & EN28 & Coste de las multas significativas y numero de sanciones no monetarias por incumplimiento de la normativa ambiental \\
\hline
\end{tabular}

- Dimensión social: En relación al desempeño social (subíndice $\mathrm{IR}_{\mathrm{s}}$ ) se establece un numero de 29 ítems (véase tabla 4), de los cuales 9 corresponden a cuestiones vinculadas con prácticas laborales y ética del trabajo (LA), 6 a derechos humanos (HR), otros 6 a cuestiones sociales (SO), a los que hay que añadir 2 incorporados por el suplemento sectorial (FS), y 4 relativos a responsabilidad sobre productos (PR), a los que hay que añadir otros 2 incorporados por el suplemento.

Por lo tanto, en total se emplean 65 ítems para analizar el comportamiento que tienen las entidades financieras españolas a la hora de divulgar información sobre RSC. Para puntuar los ítems nos basaremos en un sistema dicotómico en el que se dará un punto si la entidad incluye entre su información divulgada el ítem y cero en caso contrario. Esto sig- nifica que la sumatoria de ir será igual al número total de ítems sobre los que informe una entidad determinada.

Por último, el índice de responsabilidad agregado (IR), compuesto por los cuatro subíndices entre los que se han dividido los ítems, sería:

$$
I R=\frac{\sum_{i=1}^{65} i r_{i}}{65} \times 100
$$

La segunda parte del estudio comprende el análisis explicativo, con el que se pretende identificar aquellos factores que influyen de modo significativo en la divulgación de información sobre RSC en las entidades financieras. Para ello, a partir de una revisión de la literatura y teniendo en cuenta las caracterís-

3 Elaboración propia a partir de GRI (2006). 
Tabla 4. Ítems del desempeño social ${ }^{4}$

\begin{tabular}{|c|c|c|}
\hline $\mathbf{i}$ & Cód. & Información a divulgar \\
\hline 1 & LA1 & Desglose del colectivo de trabajadores por tipo de empleo, contrato y región \\
\hline 2 & LA2 & Número total de empleados y rotación media de empleados, desglosados por grupos \\
\hline 3 & LA4 & Porcentaje de empleados cubiertos por un convenio colectivo \\
\hline 4 & LA5 & $\begin{array}{l}\text { Periodo mínimo de preaviso relativo a cambios organizativos, incluyendo si estas notificaciones son especificadas en los convenios } \\
\text { colectivos }\end{array}$ \\
\hline 5 & LA7 & Tasas de absentismo, enfermedades profesionales, días perdidos y numero de víctimas mortales relacionadas con el trabajo por región \\
\hline 6 & LA8 & $\begin{array}{l}\text { Programas de educación, formación, asesoramiento, prevención y control de riesgos que se apliquen a los trabajadores, a sus familias } \\
\text { o a los miembros de la comunidad }\end{array}$ \\
\hline 7 & LA10 & Promedio de horas de formación al ano por empleado, desglosado por categorías \\
\hline 8 & LA13 & Composición de los órganos de gobierno corporativo y plantilla \\
\hline 9 & LA14 & Relación entre salario base de los hombres con respecto al de las mujeres \\
\hline 10 & HR1 & $\begin{array}{l}\text { Porcentaje y número total de acuerdos de inversión significativos que incluyan clausulas de derechos humanos o que hayan sido objeto } \\
\text { de análisis en materia de derechos humanos }\end{array}$ \\
\hline 11 & HR2 & $\begin{array}{l}\text { Porcentaje de los principales distribuidores y contratistas que han sido objeto de análisis en materia de derechos humanos, y medidas } \\
\text { adoptadas como consecuencia }\end{array}$ \\
\hline 12 & HR4 & Número total de incidentes de discriminación y medidas adoptadas \\
\hline 13 & HR5 & $\begin{array}{l}\text { Actividades de la compañía en las que el derecho a libertad de asociación y de acogerse a convenios colectivos puedan correr impor- } \\
\text { tantes riesgos, y medidas adoptadas }\end{array}$ \\
\hline 14 & HR6 & $\begin{array}{l}\text { Actividades identificadas que conllevan un riesgo potencial de incidentes de explotación infantil, y medidas adoptadas para contribuir } \\
\text { a su eliminación }\end{array}$ \\
\hline 15 & HR7 & Operaciones identificadas como de riesgo significativo de ser origen de trabajo forzado \\
\hline 16 & S01 & $\begin{array}{l}\text { Naturaleza, alcance y efectividad de programas y prácticas para evaluar y gestionar los impactos de las operaciones en las comuni- } \\
\text { dades }\end{array}$ \\
\hline 17 & FS13 & Puntos de acceso, por tipo, en aéreas de escasa densidad de población o desfavorecidas \\
\hline 18 & FS14 & Iniciativas para mejorar el acceso a los servicios financieros a personas desfavorecidas \\
\hline 19 & S02 & Porcentaje y número total de unidades de negocio analizadas con respecto a riesgos relacionados con la corrupción \\
\hline 20 & S03 & Porcentaje de empleados formados en las políticas y procedimientos anti-corrupción \\
\hline 21 & S04 & Medidas tomadas en respuesta a incidentes de corrupción \\
\hline 22 & S05 & Posición en las políticas públicas y participación en el desarrollo de las mismas y de actividades de $\square$ lobbying $\square$ \\
\hline 23 & S08 & $\begin{array}{l}\text { Valor monetario de sanciones y multas significativas y número total de sanciones no monetarias derivadas del incumplimiento de } \\
\text { leyes y regulaciones }\end{array}$ \\
\hline 24 & FS15 & Descripción de políticas para el correcto diseño y oferta de servicios y productos financieros \\
\hline 25 & PR1 & $\begin{array}{l}\text { Fases del ciclo de vida de los productos y servicios en las que se evalúan los impactos de los mismos en la salud y seguridad de los } \\
\text { clientes }\end{array}$ \\
\hline 26 & PR3 & $\begin{array}{l}\text { Tipos de información sobre los productos y servicios que son requeridos por los procedimientos en vigor y la normativa, y porcentaje } \\
\text { de productos y servicios sujetos }\end{array}$ \\
\hline 27 & FS16 & Iniciativas para mejorar la alfabetización y educación financiera según el tipo de beneficiario \\
\hline 28 & PR6 & Programas de cumplimiento de las leyes o adhesión a estándares y códigos voluntarios mencionados en comunicaciones de marketing \\
\hline 29 & PR9 & $\begin{array}{l}\text { Coste de aquellas multas significativas fruto del incumplimiento de la normativa en relación con el suministro y uso de productos y } \\
\text { servicios }\end{array}$ \\
\hline
\end{tabular}

4 Elaboración propia a partir de GRI (2008) y de FSSS (2008). 
ticas propias del sector, se realizará una selección de los factores que determinan que las entidades financieras divulguen más o menos información sobre RSC. A continuación, se analizaran los niveles de correlación entre las variables independientes y, finalmente, se llevará a cabo un análisis de regresión lineal múltiple, tomando como variable dependiente el índice de responsabilidad agregado (IR) calculado anteriormente.
Respecto a la revisión de la literatura, al igual que el concepto de RSC ha ido evolucionando a lo largo de las últimas décadas, los trabajos en torno a este tema también han ido incrementándose. Según Milne \& Gray (2007), la revelación de información social y medioambiental se ha manifestado como un fenómeno de los países desarrollados (principalmente Estados Unidos, Canadá y Europa), lo cual se puede corroborar analizando los principales estudios en este ámbito (ver tabla 5).

Tabla 5. Principales trabajos sobre divulgación de información sostenible ${ }^{5}$

\begin{tabular}{|c|c|c|}
\hline Autores & Año & Ámbito de estudio \\
\hline Aldamiz-Echevarría \& Aguirre & 2010 & País Vasco (España) \\
\hline Aranguren & 2008 & Alemania, España y Reino Unido \\
\hline Aranguren \& Maldonado & 2009 & México, Chile y Colombia \\
\hline Archel & 2003 & España \\
\hline Archel \& Lizarraga & 2001 & Empresas cotizadas de España \\
\hline Azcárate \& Pérez & 2001 & $\begin{array}{l}\text { Empresas del sector de abastecimiento y saneamiento de } \\
\text { agua de Andalucía y Cataluña (España) }\end{array}$ \\
\hline Bewley \& Li & 2000 & Empresas manufactureras de Canadá \\
\hline Castelo \& Lima & 2006 & Europa \\
\hline Cormier, Magnan \& Van Velthoven & 2005 & Europa \\
\hline Cowen, Ferreri \& Parker & 1987 & Estados Unidos \\
\hline Deegan, Rankin \& Tobin & 2002 & Australia \\
\hline Deegan \& Gordon & 1996 & Australia \\
\hline García-Ayuso \& Larrinaga & 2003 & España \\
\hline Gray, Kouhy \& Lavers & 1995 & Europa \\
\hline Hackston \& Milne & 1996 & Nueva Zelanda \\
\hline Maclaren & 1996 & Norteamérica \\
\hline Moneva \& Llena & 2000 & España \\
\hline Moneva, Pascual \& Fuertes & 1998 & Empresas del sector eléctrico de España \\
\hline Neu, Warsame \& Pedwell & 1998 & Canadá \\
\hline Ochoa \& Aranguren & 2005 & Empresas del IBEX35 de España \\
\hline Patten & 1991 & Estados Unidos \\
\hline Patten & 1995 & Estados Unidos \\
\hline Piechocki & 2004 & Norteamérica \\
\hline Senise et al. & 2008 & Cooperativas oleícolas de Jaén (España) \\
\hline Waller \& Lanis & 2007 & Empresas de entretenimiento norteamericanas \\
\hline Zeghal \& Ahmed & 1990 & Canadá \\
\hline
\end{tabular}

\footnotetext{
5 Elaboración propia.
} 
Por tanto, teniendo en cuenta los trabajos más representativos sobre divulgación de información sostenible hemos considerado analizar la influencia de los siguientes determinantes:

- Tamaño: De acuerdo con Firth (1979), la relevancia del factor tamaño radica en que la elaboración y difusión de información adicional implica incurrir en costes mas difíciles de afrontar para las pequeñas empresas. Por su parte, Giner (1997) explica la relación entre tamaño y revelación de información en términos de coste de agencia, de forma que para reducirlos se recurre a aumentar los niveles informativos mínimos.

Con frecuencia, en la mayoría de los trabajos se toma como medida del tamaño de la organización el total de activo, la cifra de negocios o el número de accionistas. Para el estudio que nos ocupa y dadas las características de las organizaciones analizadas se tomara como medida de tamaño el activo total.

- Cotización en el mercado de valores: Dado que la información requerida por los organismos reguladores encargados de supervisar e inspeccionar los mercados de valores, en España es la Comisión Nacional del Mercado de Valores (CNMV), es más elevada que en el caso de las empresas que no cotizan, las compañías cotizadas tienden a divulgar un mayor volumen de información (Gutiérrez \& Navallas, 2008). Por ello, se considera oportuno escoger como variable explicativa el hecho de que las entidades estudiadas coticen o no en el mercado de valores.

- Rentabilidad financiera: En base a la teoría de la agencia, los administradores tienden a revelar un mayor volumen de información cuando los indicadores que definen la posición económico -financiera de la firma son positivos (Archel \& Lizarraga, 2001). Adicionalmente, autores como Belkaoui \& Kahl (1978) sugieren que las empresas con buenos indicadores económico -financieros serán mas propensas a elaborar todo tipo de información voluntaria ya que cuentan con más recursos que las financieramente débiles o mal posicionadas. Para medir la rentabilidad financiera se utilizará el ratio $\mathrm{RE} / \mathrm{FP}$ (resultado del ejercicio sobre fondos propios).

- Endeudamiento: De acuerdo con Chow \& Wong-Boren (1987), los costes de agencia son superiores para las empresas con una mayor presencia de deuda a largo plazo en el pasivo, de forma que puede esperarse una relación directa entre el endeudamiento de la sociedad y la presentación de información voluntaria. Sin embargo, pocos trabajos han encontrado una relación directa significativa entre ambas variables, uno de ellos es el trabajo de Hossain, Perera \& Rahman (1995) que estudia la vinculación entre ambas variables en empresas cotizadas de Nueva Zelanda.

La medición del nivel de endeudamiento se llevara a cabo mediante el ratio $(P C+P N C) /$ $(P N+P C+P N C)$, es decir, pasivo corriente mas pasivo no corriente sobre pasivo corriente, no corriente y patrimonio neto.

- Tipo de entidad: Las entidades de crédito se dividen en tres tipos, en función de cuál sea su forma jurídica (bancos, cajas de ahorro o cooperativas de crédito). Así, aunque el sector de actividad para los tres tipos de entidad es el mismo, no ocurre esto con su forma jurídica y, por ende, con su finalidad social.

En el caso de los bancos, su finalidad social es similar a la de cualquier sociedad anónima. Por su parte, las cajas de ahorro, con forma jurídica de fundación privada y fines sociales, son una figura atípica en el sistema financiero. Por último, las cooperativas de crédito mantienen la forma jurídica de una sociedad cooperativa, cuyo objetivo social es servir a las necesidades financieras de sus socios y de terceros. Esta serie de contrastes hace que la inclusión del tipo de entidad como variable explicativa para el modelo resulte de interés.

- Aplicación del marco de recomendaciones GRI3: En la actualidad, la aplicación del marco de recomendaciones GRI3 para la elaboración de memorias sobre RSC es considerada como 
una garantía mínima en cuestiones como la estructura, contenido, calidad y utilidad de la información reportada mediante los informes. Dado que no todas las entidades divulgan información de acuerdo a la GRI3, ya que su aplicación es voluntaria, se ha considerado interesante distinguir unas entidades de otras.

\section{4. Ámbito de estudio}

De las distintas figuras que componen el sistema financiero español, el estudio se va a centrar en las entidades de crédito, es decir, en bancos, cajas de ahorro o cooperativas de crédito. La muestra de entidades ha sido escogida atendiendo a la disponibilidad online de su memoria o informe de RSC/sostenibilidad y de sus cuentas anuales. En ambos casos dichas memorias e informes, a efectos de paridad comparativa del estudio entre entidades, se corresponden al ejercicio 2010.

La selección de las entidades ha sido confeccionada a partir de la información que ofrece el Registro de Entidades online del Banco de España y la Global Reporting Iniciative. Así, considerando tanto las entidades nacionales como las filiales extranjeras con forma jurídica nacional, en 2010 operaban en España 64 bancos, 46 cajas de ahorro y 83 cooperativas de crédito (Banco de España, 2011).

Respecto a los bancos, solo 9 presentan memorias o informes online relacionados con RSC, de los que 6 siguen las recomendaciones de la GRI3. En cuanto a las cajas de ahorro, 17 de las 18 cajas seleccionadas aplican en sus memorias las recomendaciones de la GRI3. En último lugar, se han seleccionado únicamente 7 cooperativas de crédito, de las que 4 siguen la GRI3.

Por tanto, la muestra definitiva está compuesta por 34 entidades de crédito, de ellas 27 estructuran sus memorias de RSC conforme a la GRI3 mientras que las 7 restantes elaboran sus informes de sostenibilidad de acuerdo a sus propios criterios.

\section{Divulgación sobre RSC en el sector financiero}

\subsection{Análisis descriptivo}

En primer lugar se han estimado los diferentes subíndices sobre la divulgación de información para cada una de las dimensiones estudiadas (impacto de productos y servicios, económica, ambiental y social). Así, como se puede observar en el Ilustracion 1, la dimensión sobre la que mayor volumen de información se reporta es la económica, seguida por la de carácter social; siendo divulgados en las memorias e informes analizados un $81,93 \%$ de los ítems seleccionados para el primer caso y un $76,77 \%$ en el segundo.

También se aprecia como la información relativa al impacto de productos y servicios, que es de carácter especifico para el sector (GRI, 2008), es la que menos tienden a divulgar las entidades de la muestra $(64,71 \%)$. Por su parte, la dimensión relacionada con los aspectos medioambientales es atendida en el $72,15 \%$ de los casos.

En relación al índice de responsabilidad agregado (IR), compuesto por estos cuatro subíndices, los resultados indican que el $73,89 \%$ de los ítems estudiados son divulgados en las memorias de las entidades de la muestra.

Ilustración 1. Divulgación de RSC por dimensiones ${ }^{6}$

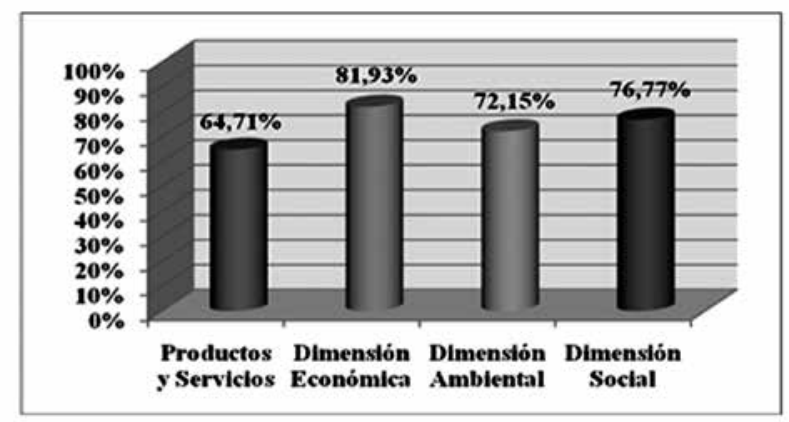

$6 \quad$ Elaboración propia. 
A continuación, se analiza cada uno de los subíndices utilizados con el objetivo de comprobar cuales son los factores de mayor relevancia para las entidades de este sector a la hora de divulgar información sobre RSC.

Respecto al subíndice de carácter especifico para el sector de los servicios financieros (Productos y Servicios), compuesto de 12 ítems (véase Ilustracion 2), encontramos que el indicador FS1, relativo a las políticas medioambientales y sociales aplicadas a las líneas de negocio, es el ítem empleado con mayor frecuencia por las entidades analizadas $(79,41 \%)$.En segundo lugar aparecen, con volúmenes superiores al 70\%, los ítems relacionados con los procesos para mejorar las competencias de los empleados para implementar políticas y procedimientos medioambientales y sociales (FS4), las interacciones con clientes o sociedades participadas en relación a los riesgos y oportunidades medioambientales y sociales (FS5) y el valor monetario de productos y servicios diseñados para el beneficio social (FS7).

En la cara opuesta en cuanto a frecuencia de divulgación, se encuentra aquella información vinculada a los procesos para monitorizar la implementación por parte de los clientes de los requisitos legales y medioambientales incluidos en contratos o transacciones (FS3) y la relativa a la cobertura y frecuencia de auditorías para evaluar la implementación de las políticas medioambientales y sociales y los riesgos (FS9), ambos ítems con un 52,94\%.

En la dimensión económica, compuesta por 7 ítems, es el indicador EC3, que informa sobre la cobertura de las obligaciones de las entidades vinculadas a programas de beneficios sociales, del que con mayor asiduidad se informa, alcanzando un porcentaje superior al $90 \%$. Le siguen el indicador referido al valor económico directo generado y distribuido (EC1), con un $91,18 \%$, y el de desarrollo e impacto de las inversiones en infraestructuras y de servicios prestados (EC8), con un $82,35 \%$.

La información relativa a ayudas financieras recibidas de gobiernos (EC4) es la que en menor número de ocasiones se encuentra en los informes de las entidades que constituyen la muestra.
La dimensión ambiental, explicada en este estudio mediante 17 ítems, contiene la única información que divulgan todas las entidades que conforman la muestra, independientemente de si siguen la GRI3 o no. Este ítem, el indicador EN26, informa sobre las iniciativas que se llevan a cabo con el fin de mitigar los impactos ambientales de productos y servicios.

Los indicadores EN1 y EN28, vinculados a la divulgación de información sobre materiales utilizados y coste de multas y número de sanciones por incumplimiento de la normativa ambiental, respectivamente; superan el $80 \%$ de casos en los que la información era presentada en el informe. En, prácticamente, el $80 \%$ de los informes analizados se trataban aspectos vinculados con el porcentaje de materiales utilizados que son valorizados (EN2), con el consumo directo de energía desglosado por fuentes primarias (EN3) o sobre el peso total de los residuos gestionados (EN22). No obstante, tan solo la mitad de los informes contenían información relativa a productos vendidos recuperados al final de su vida útil (EN27). Aunque, tratándose de entidades cuyos productos no son, en su mayoría, materiales, se aprecia el esfuerzo de algunas de ellas por cumplir con la información a divulgar recomendada, aportando la información que les es posible.

En relación al ámbito del desempeño social, compuesto de 29 ítems repartidos en 4 categorías, laboral (LA) representada mediante 8 indicadores, derechos humanos (HR) con 6, social (SO) con 8 (de los cuales 2 son específicos del sector (FS)) y productos con 6 (también con 2 específicos); se observa como el ítem relacionado con la divulgación de información sobre programas de educación, formación, asesoramiento, prevención y control de riesgos (LA8) es el que con mayor frecuencia se reporta $(94,12 \%)$.

También, la información relativa al porcentaje de empleados cubiertos por un convenio colectivo $(88,24 \%)$, indicador LA4, se proporciona habitualmente en las memorias. En ambos casos se trata de indicadores de índole laboral.

En lo referente a los derechos humanos, destaca la frecuencia del ítem HR4 relativo a cuestiones vinculadas a la discriminación, que aparece en el $82 \%$ de los in- 
formes. Entre estos, también se encuentra el ítem con menor presencia, tanto en la dimensión social como entre el conjunto de dimensiones, con poco más de un $30 \%$ de casos en los que se informa sobre el porcentaje de principales distribuidores y contratistas (HR2).

Entre los indicadores de índole puramente social, destacan por su número de apariciones $(82,35 \%)$ los relativos a las medidas tomadas ante incidentes de corrupción y sanciones (SO4) y a multas derivadas del incumplimiento de las leyes y regulaciones (SO8). Aunque, no ocurre lo mismo con la información relativa a la posición ante políticas publicas y actividades de "lobbying" (SO5) que se encuentra en menos del $68 \%$ de las memorias.

Por último, en relación a la responsabilidad sobre productos, destaca la información divulgada en torno a programas de cumplimiento de leyes o adhesión a estándares y códigos voluntarios (PR6) que se cubre en más del $85 \%$ de los informes. Sin embargo, la información referida a las fases del ciclo de vida de los productos y servicios en las que se evalúan los impactos de los mismos en la salud y seguridad de los clientes (PR1) no se encuentra disponible más que en el $67 \%$ de los informes.

Tabla 6. Matriz de correlaciones ${ }^{7}$

\begin{tabular}{|c|c|c|c|c|c|c|c|c|}
\hline & & \multirow{3}{*}{$\begin{array}{c}\mathbf{I R} \\
1\end{array}$} & & & & & & \\
\hline & & & Tam & Cot & Ren & End & \multirow[t]{2}{*}{ Tip } & \multirow[t]{2}{*}{ Apl } \\
\hline \multirow{3}{*}{$\mathbb{R}$} & Correlación de Pearson & & & & & & & \\
\hline & Sig. (bilateral) & & & & & & & \\
\hline & $\mathrm{N}$ & 34 & & & & & & \\
\hline \multirow{3}{*}{ Tam } & Correlación de Pearson & 119 & 1 & & & & & \\
\hline & Sig. (bilateral) &, 501 & & & & & & \\
\hline & $\mathrm{N}$ & 34 & 34 & & & & & \\
\hline \multirow{3}{*}{ Cot } & Correlación de Pearson & ,223 &, $488^{\star \star \star}$ & 1 & & & & \\
\hline & Sig. (bilateral) & ,206 & ,003 & & & & & \\
\hline & $\mathrm{N}$ & 34 & 34 & 34 & & & & \\
\hline \multirow{3}{*}{ Ren } & Correlación de Pearson &,- 014 &, $532^{\star \star \star}$ & $658^{\star \star \star \star}$ & 1 & & & \\
\hline & Sig. (bilateral) & 936 & 001 & 000 & & & & \\
\hline & N & 34 & 34 & 34 & 34 & & & \\
\hline \multirow{3}{*}{ End } & Correlación de Pearson &,- 235 & ,084 & ,241 &, 129 & 1 & & \\
\hline & Sig. (bilateral) & , 180 & ,635 & ,169 & ,468 & & & \\
\hline & $N$ & 34 & 34 & 34 & 34 & 34 & & \\
\hline \multirow{3}{*}{ Tip } & Correlación de Pearson &, $471^{\star \star *}$ &,- 338 &,- 499 *** &,$- 481^{* \star *}$ &,- 213 & 1 & \\
\hline & Sig. (bilateral) & 005 & 051 & ,003 & 004 & ,226 & & \\
\hline & $\mathrm{N}$ & 34 & 34 & 34 & 34 & 34 & 34 & \\
\hline \multirow{3}{*}{ Apl } & Correlación de Pearson &, $881^{\star * *}$ & ,201 & ,282 & , 080 &,- 239 &, 329 & 1 \\
\hline & Sig. (bilateral) &, 000 & ,253 &, 106 & 651 &, 173 & 057 & \\
\hline & N & 34 & 34 & 34 & 34 & 34 & 34 & 34 \\
\hline
\end{tabular}

* La correlación es significativa al nivel 0,1 (bilateral). ** La correlación es significativa al nivel 0,05 (bilateral). *** La correlación es significativa al nivel 0,01 (bilateral).

$7 \quad$ Elaboración propia. 


\subsection{Análisis explicativo}

En primer lugar se va a analizar la matriz de correlaciones (véase tabla 6) con el fin de comprobar si existe correlación entre las variables del modelo. Para ello, se aplica el coeficiente de correlación de Pearson que mide la relación lineal entre cada una de las parejas de variables.

De la matriz de correlaciones destaca la existencia de un elevado grado de correlación entre la variable dependiente (IR) y la variable independiente referida a la aplicación del marco de recomendaciones GRI3 (Apl). A su vez, algunas de las variables independientes están correlacionadas de forma moderada o baja. Es el caso de la relación entre las variables cotización y tamaño, ya que, por lo general, son las entidades de mayor dimensión las que suelen acudir a los mercados de valores para financiarse. Pero en general, ninguna de las relaciones entre las variables explicativas toma valores que aconsejen su exclusión del modelo.

A continuación, una vez comprobadas las hipótesis de partida del modelo (linealidad, homocedasticidad, normalidad, independencia y no co-linealidad) se realiza la regresión lineal múltiple, indicando los resultados obtenidos que el modelo presenta un ajuste medio-alto (ver tabla 7), como se puede comprobar a partir del $\mathrm{R}^{2}(79,70 \%)$. En cuanto a la capacidad de influencia que tienen las variables escogidas como factores determinantes, tres de las seis escogidas son significativas.

En relación a la variable que indica si la entidad cotiza o no en un mercado de valores (Cot), es significativa a un nivel de confianza del $90 \%$ y tiene un efecto positivo sobre el modelo estimado. Es decir, que cotizar en el mercado de valores incide positivamente en el volumen de información divulgada sobre RSC. Este resultado es afín a los obtenidos por autores como Firth (1979), que señala la necesidad de las sociedades cotizadas por buscar la confianza de los inversores mediante la divulgación de mayores volúmenes de información voluntaria.
Tabla 7. Resultados de la regresión ${ }^{8}$

\begin{tabular}{|c|c|c|c|c|}
\hline \multirow{2}{*}{ Modelo } & \multicolumn{2}{|c|}{$\begin{array}{l}\text { Coeficientes no } \\
\text { estandarizados }\end{array}$} & \multirow{2}{*}{$\begin{array}{c}\begin{array}{c}\text { Coeficientes } \\
\text { tipificados }\end{array} \\
\text { Beta }\end{array}$} & \multirow{2}{*}{$\mathbf{t}$} \\
\hline & B & Error típ. & & \\
\hline (Constante) & ,704 & 1,156 & & 609 \\
\hline Tam &, 000 &, 000 & ,013 & ,138 \\
\hline Cot & ,169 &, 093 & ,236 & $1,806^{*}$ \\
\hline Ren &,- 647 &, 971 &,- 075 &,- 666 \\
\hline End &,- 682 & 1,218 &,- 048 &,- 560 \\
\hline Tip & ,112 &, 040 & ,315 & $2,781^{* * *}$ \\
\hline \multirow[t]{3}{*}{ Apl } &, 528 &, 080 & ,703 & $6,574^{* * *}$ \\
\hline & & $\mathrm{R}$ & $\mathrm{R} 2$ & $\begin{array}{c}\mathrm{R} 2 \\
\text { corregida }\end{array}$ \\
\hline & &, 913 & 834 &, 797 \\
\hline
\end{tabular}

La mayor regulación que sufren las entidades cotizadas en cuanto a divulgación de información, a través de normativa como la Ley 26/2003, de 17 de julio; cuyo fin es reforzar la transparencia de las sociedades anónimas cotizadas, conlleva al incremento de la transparencia de este tipo de compañías, aunque no tiene por qué influir directamente en la divulgación de información sobre RSC.

La variable explicativa relativa al tipo de entidad de crédito es significativa para un nivel de confianza del 99\%. Lo que indica que los diferentes objetivos y fines sociales que caracterizan a los bancos, cajas de ahorros y cooperativas de crédito son uno de los factores que determinan el grado de información divulgada sobre RSC. Tal y como se desprende del análisis descriptivo, son las cajas de ahorro el tipo de entidades que por término medio mayor volumen de información divulgan.

En cuanto a la variable referida a la aplicación del marco de recomendaciones GRI3, también resulta significativa a un nivel de confianza del $99 \%$, manteniendo una relación positiva con la variable dependiente. Por lo tanto, las entidades que aplican la GRI3 divulgan más información sobre RSC que el resto. Con ello se confirma empíricamente

8 Elaboración propia. 
la relación que ya se intuía al analizar la matriz de correlaciones.

Para el resto de variables independientes analizadas (tamaño, rentabilidad y endeudamiento), no existen evidencias suficientes que indiquen la existencia de alguna relación significativa entre dichas variables $y$ el índice de responsabilidad agregado (IR).

\section{Conclusiones}

Aunque en un principio se consideraba que las actividades llevadas a cabo por las organizaciones que operan en el sector financiero tenían un escaso impacto social y medioambiental. La reciente preocupación generalizada sobre la repercusión que las políticas asumidas por estas entidades puede tener sobre la marcha y la estabilidad económica, medioambiental y social ha sido tal que la GRI ha considerado necesario emitir una adaptación para este tipo de organizaciones mediante el Suplemento Sectorial para Servicios Financieros.

En España, a pesar de la tardía introducción del concepto de RSC, el sector financiero goza actualmente de una destacada posición entre los sectores que mayor número de memorias publican y cada vez un número mayor de entidades pertenecientes a este sector son más propensas a publicar información sobre sostenibilidad (según la Corporate Register, la base de datos sobre reportes de sostenibilidad más grande del mundo). Sin embargo, tras el escrutinio realizado durante la búsqueda de las memorias o informes para la selección de la muestra se ha comprobado como el porcentaje de entidades de crédito con forma jurídica española que divulgan un volumen de información significativa sobre RSC a través de su página web se sitúa en torno a un $18 \%$.

En general, el volumen de información divulgada sobre RSC, medido a partir de los ítems establecidos, es elevado, informando por término medio sobre, prácticamente, un $74 \%$ de los ítems. El tipo de información que con mayor frecuencia tienden a divulgar sobre RSC tiene que ver con aspectos económicos. Este hecho puede ser fruto del menor coste que supone la búsqueda y elaboración de información e indicadores de este tipo, debido a que los datos necesarios para hacerlo pueden estar disponibles al tener que cumplir con algunos requisitos legales, o bien, se desprenden de otros informes ya elaborados.

La información relacionada con las materias social y medioambiental, es por este orden, la que más se divulga tras los aspectos económicos. Destaca, significativamente, el hecho de que en todas las memorias sobre RSC analizadas se informe acerca de las iniciativas para mitigar los impactos ambientales de los productos y servicios, ítem de carácter medioambiental seleccionado para el estudio.

Por su parte, la información sobre el impacto de productos y servicios es la que con menor asiduidad se divulga, probablemente porque el coste de su elaboración unido a la especificidad de su contenido disuade a las entidades. Destaca el volumen de información que divulgan las cajas de ahorro, ya que reportan casi el $90 \%$ de los ítems. Coincidiendo con la opinión de algunas publicaciones como la de Quintas (2006), se comprueba como las cajas de ahorro van a la vanguardia en la integración de la RSC asumiéndola en su razón de ser, ya que esta se encuentra inscrita en sus principios fundacionales.

Por otro lado, tras estudiar los principales trabajos explicativos sobre divulgación de información sostenible se han observado notables diferencias en los resultados y las conclusiones, que pueden ser debidas a la metodología aplicada, el tipo de sector analizado, el año de realización del estudio o la localización geográfica de la muestra. En nuestro caso, las variables independientes analizadas que explican la divulgación de información sobre RSC en el sector financiero español son la cotización en el mercado de valores, el tipo de entidad de crédito y la aplicación del marco de recomendaciones GRI3.

La razones por las que el hecho de cotizar en un mercado de valores influye positivamente en la información divulgada sobre RSC son variadas. Por un lado, se puede justificar mediante la presión hacia la transparencia que sufren las sociedades cotizadas por parte de los organismos reguladores, aunque tal presión no conduzca, al menos de manera explícita, al suministro de información voluntaria sobre RSC. Por otro lado, puede deberse a una posible estrategia de las empre- 
sas cotizadas consistente en aumentar la divulgación de información sobre sostenibilidad con objeto de incrementar la confianza de los inversores y reducir los riesgos que afectan al precio de sus títulos.

La relación del volumen de información aportada con las variables tipo de entidad y aplicación de la GRI3 queda contrastada, en el caso de la primera, por el comportamiento diferenciador de las cajas de ahorro anteriormente citado, y en el segundo, por la ausencia de la aplicación de otros marcos de referencia en aquellas memorias que no siguen esta guía, y que por lo tanto no suelen aportar información útil y precisa.

Por tanto, como resumen de las principales aportaciones de este estudio a la literatura existente sobre $\mathrm{RSC}$, se puede señalar que, a pesar de la buena reputación del sector financiero español en la elaboración de memorias de sostenibilidad, el porcentaje de las entidades analizadas que divulgan información de este tipo es escaso. Sin embargo, el contenido de los informes analizados es satisfactorio, sobre todo en el caso de las cajas de ahorro, el volumen y la calidad de información divulgada es elevado, destacando la de tipo económico. Respecto a los determinantes del suministro de información sostenible en las entidades de crédito españolas, la cotización en el mercado de valores, el tipo de entidad de crédito y la aplicación del marco de recomendaciones GRI3 han resultado significativos.

Entre las principales limitaciones con las que cuenta este estudio se encuentra la del acceso a la información divulgada, ya que únicamente se han podido analizar aquellas entidades que publican los informes en su página web corporativa. Por lo tanto, en estudios posteriores sería conveniente establecer contactos con las entidades para averiguar si elaboran memorias de RSC e incluirlas en la muestra.

Como futuras líneas de investigación, se podría analizar la evolución de la divulgación de información sobre RSC en el sector. Por otro lado, también sería interesante ampliar la muestra seleccionando entidades financieras de otros países, lo que permitiría realizar comparativas entre ellos.

\section{Referencias}

AECA (2004) Marco Conceptual de la Responsabilidad Social Corporativa. Comisión de Responsabilidad Social Corporativa.

Albareda, L. \& Balaguer, M.R. (2007). "La responsabilidad social de la empresa y los resultados financieros". Contabilidad y dirección, Barcelona.

Aldamiz-Echevarría, C. \& Aguirre, M.S. (2010) "La comunicación de la responsabilidad social de las mayores empresas vascas: un análisis comparativo". XX Jornadas hispano-lusas de gestión científica.

Aranguren, N. (2008) "Divulgación de información sobre responsabilidad social: un análisis comparativo entre empresas cotizadas de Alemania, España y Reino Unido". Directora: Elena Ochoa. Universidad de Deusto.

Aranguren, N. \& Maldonado, S. (2009) "La divulgación de información social en América Latina: El caso de México, Chile y Colombia". XV Congreso AECA: Decidir en época de crisis: transparencia y responsabilidad.

Archel, P. (2003) "La divulgación de información social y medioambiental de la gran empresa española en el período 1994-1998". En: Revista Española de Finanzas y Contabilidad, 32(117): 571-601.

Archel, P. \& Lizarraga, F. (2001) "Algunos determinantes de la información medioambiental divulgada por las empresas españolas cotizadas". En: Revista de Contabilidad, 4(7):129-153.

Azcarate, F. \& Pérez, V.A. (2001) "Estudio de la publicación de información medioambiental en empresas del sector de abastecimiento y saneamiento de agua tras la adaptación sectorial del Plan General Contable". XI Congreso AECA: Empresa, Euro y Economía.

Banco de España (2011) Consulta online del Registro Oficial de Entidades. http://app.bde.es/ren/html/inicio.htm

Belkaoui, A. \& Kahl, A. (1978) "Corporate financial disclosure in Canada". Canadian Certified General Accountants Association. Vancouver, Canada. Research monograph No 1.

Bewley, K. \& Li, Y. (2000) "Disclosure of environmental information by Canadian manufacturing companies: a voluntary disclosure perspective". En. Advances in environmental accounting and management, 1:201-226.

Castelo, M. \& Lima, L. (2006) "Communication of corporate social responsibility by Portuguese banks. A legitimacy theory perspective". En: Corporate Communications: An International Journal, 11(3):232-248.

Chow, CH. \& Wong-Boren, A. (1987) "Voluntary financial disclosure by Mexican corporations". En The Accounting Review, 62(3):533-541.

Comisión Europea (2001) Libro Verde de la Comisión, julio de 2001. DIARIO OFICIAL DE LA UNION EUROPEA, 27 de mayo de 2002, C. 125

Cormier, D., Magnan, M. \& Van Velthoven, B. (2005) "Environmental Disclosure Quality in Large German Companies: Economic Incentives, Public Pressures or Institutional Conditions?" En: European Accounting Review, 14(1):3-39.

Cowen, S., Ferreri, L. \& Parker, L.D. (1987) "The impact of corporate characteristics on social responsibility disclosure: A typology and frequency-based analysis". En: Accounting, Organizations and Society, 12(2):111-122.

Davis, K. (1973) "The case for against business assumption of social responsability"', En. Academy of Management Journal, 16(2):312-322. 


\section{LOS DETERMINANTES DE LA DIVULGACIÓN DE INFORMACIÓN SOBRE RESPONSABILIDAD SOCIAL CORPORATIVA EN EL SECTOR FINANCIERO: EL CASO ESPAÑOL}

Davis K. \& Blomstroms, R. (1975) Business and Society. Enviorment and Responsibility, McGraw-Hill, Nueva York.

Deegan, C. \& Gordon, B. (1996) "A study of the environmental disclosure practices of Australian corporations". Accounting and Business Research, 26(3):187-199.

Deegan, C., Rankin, M. \& Tobin, J. (2002) "An examination of the corporate social and environmental disclosures of BHP from 1983-1997. A test of legitimacy theory". En: Accounting, Auditing and Accountability Journal, 15(3): 312-343.

De La Cuesta, M. (2006) "Responsabilidad social en el sector bancario. Su contribución a un desarrollo más sostenible". En: Papeles de Economía Española. (108):173-190.

Firth, M. (1979) "The impact of size, stock market listing and auditors on voluntary disclosure in corporate annual reports". En: Accounting \& Business Research, Autumn: 273-280.

García-Ayuso, M. \& Larrinaga, C. (2003) "Environmental disclosure in Spain: Corporate characteristics and media exposure". En: Revista Española de Finanzas y Contabilidad, (115): 184-214.

Giner, B. (1997) "The influence of company characteristics and accounting regulation on information disclosed by spanish firms". En: European Accounting Review. 6(1): 45-68.

Global reporting initiative (2006) Guía para la Elaboración de Memorias de Sostenibilidad.

Global reporting initiative (2008) Guía para la Elaboración de Memorias de Sostenibilidad. Suplemento Sectorial para los Servicios Financieros.

Gray, R., Kouhy, R. \& Lavers, S. (1995) "Corporate social and environmental reporting: a review of the literature and a longitudinal study of UK disclosure". En: Accounting, Auditing \& Accountability Journal, 8(2):47-77.

Gutierrez, H. \& Navallas, B. (2008) "Divulgación voluntaria de información: Estado de la cuestión". En: Partida Doble, (195):64-75.

Hackston, D. \& Milne, M.J. (1996) "Some determinants of social environmental disclosures in New Zealand companies". En: Accounting, Auditing and Accountability Journal, 9(1):77-95.

Hossain, M., Perera, H.B. \& Rahman, A.R. (1995) "Voluntary disclosure in the annual reports of New Zealand companies". Journal of International Financial Management \& Accounting, 6(1):69-87.

Larrinaga, C.; Moneva, J.M.; Llena, F.; Carrasco, F. \& Correa, C. (2002) Regulación contable de la información medioambiental. Normativa española e internacional, AECA, Madrid.

Ley 26/2003, de 17 de julio, por la que se modifican la Ley 24/1988 y el Real Decreto Legislativo 1564/1989, con el fin de reforzar la transparencia de las sociedades anónimas cotizadas. Boletín Oficial del Estado, núm. 171 de 18 de julio de 2003, pp. 28046-28052.
Maclaren N. (1996) "Urban Sustainability Reporting". En: Journal of the American Planning Association, 62(2):184-202.

McGuire, J.W. (1963) Bussines and Society. McGraw-Hill, Nueva York.

Milne, M.J. \& Gray, R.H. (2007) "The Future of Sustainability Reporting, chapter 10", en Unerman J., O'Dwyer, B. y Bebbington, J. (eds.), Sustainability, Accounting and Accountability, Routledge: London.

Moneva, J.M., Pascual, E. \& Fuertes, Y. (1998) "Información financiera medioambiental en el sector eléctrico". En: Actualidad financiera, 3(4).

Moneva, J.M. \& Llena, F. (2000) "Environmental disclosures in the annual reports of large companies in Spain". En: European Accounting Review, (9):7-29.

Navarro, F. (2008) Responsabilidad Social Corporativa: Teoría y práctica. ESIC, Madrid.

Neu, D., Warsame, H. \& Pedwell, K. (1998) "Managing public impressions: environmental disclosures in annual reports". En: Accounting, Organizations and Society, 23(3): 265-282.

Ochoa, E. \& Aranguren, N. (2005) "Divulgación de información social y medioambiental. Un estudio empírico de las empresas del IBEX35". XIII Congreso AECA: Armonización y gobierno de la diversidad.

Patten, D.M. (1991) "Exposure, legitimacy, and social disclosure". En: Journal of Accounting and Public Policy, (10):297-308.

Patten, D.M. (1995) "Variability in social disclosure: a legitimacybased analysis". En: Advances in Public Interest Accounting, (6):273-285

Piechocki, R. (2004) "Transparency of Annual Sustainability Reports". En: Corporate Reputation Review, 7(2):107-123.

Quintas, J. (2006) "Las cajas de ahorros en el ámbito de la responsabilidad social corporativa". En: Papeles de Economía Española, (108):128-143.

Senise, O., Torres, F.J., Parras, M. \& Murgado, E.M. (2008) "Factores inductores de un comportamiento medioambientalmente sostenible: el caso de las cooperativas oleícolas giennenses". En: Revista de Economía Pública, Social y Cooperativa, (61): 157-177.

Tostes, M. (2008) "Estudios sobre responsabilidad social en el sector financiero de Perú y Brasil: Análisis comparativo". Red Puentes, serie de estudios - Grupo financiero № 3.

Waller D. \& Lanis, R. (2007) "Corporate Social Responsibility Disclosure: An Exploratory Study of the Top 10 Media Organizations". ANZMAC Conference: 3Rs Reputation, Responsibility \& Relevance - the role of marketing in the future.

Zeghal, D. \& Ahmed, S.A. (1990) "Comparison of social responsibility information disclosure media used by Canadian firms", En: Accounting, Auditing and Accountability Journal, 3(1):38-53. 